developers carries the risk of collaboration problems, but when managed successfully, adds value to the end product.

Implications for Guideline Developers/Users In order to avoid collaboration problems, the scope of the guideline was agreed on in an early stage. Interdisciplinary collaboration enhanced guideline quality.

\section{P055 DOES THE CANADIAN TASK FORCE ON PREVENTIVE HEALTH CARE MEET THE INSTITUTE OF MEDICINE STANDARDS IN GUIDELINE DEVELOPMENT?}

${ }^{1} \mathrm{~S}$ Gorber, ${ }^{2} \mathrm{~A}$ Jaramillo, ${ }^{3} \mathrm{~K}$ Pottie, ${ }^{4} \mathrm{H}$ Singh. ${ }^{1}$ Public Health Agency of Canada, Ottawa, Canada; ${ }^{2}$ Public Health Agency of Canada, Ottawa, Canada; ${ }^{3}$ University of Ottawa, Ottawa, Canada; ${ }^{4}$ University of Manitoba, Winnipeg, Canada

\section{0:1136/bmjqs-2013-002293.141}

Background There are a plethora of guideline development groups in operation, each with its own methods for summarising evidence and processes for developing recommendations, which can leave consumers with questions about which guidelines to follow. In 2011 the Institute of Medicine (IOM) released a set of standards for developing trustworthy systematic reviews and clinical practice guidelines (CPGs). These standards address the structure, process, reporting and final products associated with evidence-based CPGs. The Canadian Task Force on Preventive Health Care (CTFPHC) was recently re-established with a mandate to develop clinical practice guidelines for primary care practitioners based on a systematic assessment of scientific evidence.

Objectives To compare the Canadian Task Force on Preventive Health Care's methods with the standards outlined in the IOM.

Methods The methods of the CTFPHC were compared to the IOM standards for both systematic reviews and CPGs and to the methods of other international guideline producers.

Results The CTFPHC methods are consistent with those of the IOM and international guideline development groups. Some differences include how patient and consumer input is incorporated into guidelines, the review of documents, and the final recommendation statements, the degree to which documents are publicly available and the processes for dissemination and knowledge translation.

Discussion New processes put in place to address the differences between CTFPHC methods and IOM standards will be explored.

Implications for Guideline Developers/Users Comparing the methods of guideline development groups to the IOM standards may provide users with a way to ascertain potential areas for enhancement of their CPG development process.

\section{P056 THE CANADIAN TASK FORCE ON PREVENTIVE HEALTH CARE: INTERPRETATION TOOL TO COMPARE PREVIOUS GRADING OF RECOMMENDATIONS TO GRADE}

L Dunfield, S Gorber, A Shane. Public Health Agency of Canada, Ottawa, Canada

10:1136/bmjqs-2013-002293.142

Background Guideline producers use various methods to grade their recommendations. The Canadian Task Force on Preventive Health Care (CTFPHC) previously assigned letter grades to their recommendations based on an evaluation of the evidence considering only study design. The CTFPHC now uses GRADE (Grading of Recommendations Assessment, Development and Evaluation), which considers the quality of the evidence and the strength of the recommendations.

Objectives The objectives were to develop a tool that would allow interpretation of the previous CTFPHC grading system to GRADE to enable comparison of guidelines.

Methods A comparison and mapping of each level of evidence and strength of recommendation for each grading system was undertaken. The methods working group and the knowledge translation working group of the CTFPHC reviewed the mapping system, which was then tested with a previous CTFPHC recommendation from 2001.

Results An interpretation tool which maps the level of evidence and the strength of recommendation was created and applied to a previous CTFPHC recommendation statement.

Implications for Guideline Developers/Users The tool is not meant to be a perfect system and does not require a formal assessment of the evidence from former guidelines. The evidence from previous guidelines is not assessed using GRADE, but rather interpreted for GRADE language. This tool will be useful in helping guideline developers compare guidelines using different grading systems and will allow the CTFPHC to compare previous guidelines to GRADE. This tool may also be applied when critically appraising guidelines from other guideline development groups.

\section{P058 DEVELOPING A YOUTH HEALTH CARE GUIDELINE ON SEXUAL DEVELOPMENT WITH LIMITED EVIDENCE}

${ }^{1} \mathrm{~S}$ Maris, ${ }^{2} \mathrm{~J}$ Deurloo, ${ }^{2} \mathrm{C}$ Lanting, ${ }^{2} \mathrm{M}$ Kamphuis, I van der Vlugt. ${ }^{1}$ Rutgers WPF, Utrecht, The Netherlands; ${ }^{2}$ TNO Child Health, Leiden, The Netherlands

\section{0:1136/bmjqs-2013-002293.143}

Background Children's sexual development starts at an early age and is a very complex subject, comprising the physical but also the psychosocial development. Youth Health Care (YHC) professionals can prevent and detect problems in sexual development, and play a guiding role in stimulating sexual competency and positive sexual attitudes.

Objectives Our goal was to develop an evidence based national YHC guideline for sexual development.

Methods The content of the guideline is based on (inter)national guidelines, literature searches, consensus and experience. The guideline is now piloted for use in daily practice by YHC professionals. The way we handled the limited amount of evidence and the results of this pilot will be presented. We cooperated with an international centre of expertise on sexual and reproductive health and performed literature searches for a selected number of questions.

Results The guideline describes the (physical and psychosocial) sexual development of children from 0-19 years old, determinants of sexual health and groups at risk. Discussion In this presentation, we would like to discuss the issue of dealing with the limited amount of evidence and we will show how we handled this issue. Working together with an experienced centre was crucial. Coming to consensus in the working group and performing a pilot test in addition, is essential in gaining obtaining support for the recommendations of the guideline.

Implications for Guideline Developers/Users In YHC not much evidence of high quality is available. Exchange of experiences will help other guideline developers dealing with this as well. 